\title{
ON THE GENERALIZATION OF FALTINGS' ANNIHILATOR THEOREM
}

\author{
MOHAMMAD-REZA DOUSTIMEHR AND REZA NAGHIPOUR*
}

\begin{abstract}
Let $R$ be a commutative Noetherian ring and let $n$ be a non-negative integer. In this article, by using the theory of Gorenstein dimensions, it is shown that whenever $R$ is a homomorphic image of a Noetherian Gorenstein ring, then the invariants $\inf \left\{i \in \mathbb{N}_{0} \mid \operatorname{dim} \operatorname{Supp}\left(\mathfrak{b}^{t} H_{\mathfrak{a}}^{i}(M)\right) \geq n\right.$ for all $\left.t \in \mathbb{N}_{0}\right\}$ and $\inf \left\{\lambda_{\mathfrak{a} R_{\mathfrak{p}}}^{\mathfrak{b} R_{\mathfrak{p}}}\left(M_{\mathfrak{p}}\right) \mid \mathfrak{p} \in\right.$ Spec $R$ and $\operatorname{dim} R / \mathfrak{p} \geq n\}$ are equal, for every finitely generated $R$-module $M$ and for every ideals $\mathfrak{a}, \mathfrak{b}$ of $R$ with $\mathfrak{b} \subseteq \mathfrak{a}$. This generalizes the Faltings' Annihilator Theorem [G. Faltings, Über die Annulatoren lokaler Kohomologiegruppen, Arch. Math. 30 (1978) $473-476]$.
\end{abstract}

\section{INTRODUCTION}

Throughout this paper, let $R$ denote a commutative Noetherian ring (with identity) and $\mathfrak{a}$ an ideal of $R$. For an $R$-module $M$, the $i$ th local cohomology module of $M$ with support in $\operatorname{Var}(\mathfrak{a})$ is defined as:

$$
H_{\mathfrak{a}}^{i}(M)=\underset{n \geq 1}{\lim _{n \geq 1}} \operatorname{Ext}_{\mathrm{R}}^{\mathrm{i}}\left(\mathrm{R} / \mathfrak{a}^{\mathrm{n}}, \mathrm{M}\right) .
$$

Local cohomology was first defined and studied by Grothendieck. We refer the reader to [4] or [8] for more details about local cohomology. An important theorem in local cohomology is Faltings' Annihilator Theorem [6] for local cohomology modules, which states that, if $R$ is a homomorphic image of a regular ring or $R$ has a dualizing complex, then the invariants $f_{\mathfrak{a}}^{\mathfrak{b}}(M)$ and $\lambda_{\mathfrak{a}}^{\mathfrak{b}}(M)$ are equal, for every choice of the finitely generated $R$-module $M$ and for every choice of the ideals $\mathfrak{a}, \mathfrak{b}$ of $R$ with $\mathfrak{b} \subseteq \mathfrak{a}$, where $f_{\mathfrak{a}}^{\mathfrak{b}}(M)=\inf \left\{i \in \mathbb{N}_{0} \mid \mathfrak{b} \nsubseteq\right.$ $\left.\operatorname{Rad}\left(0: H_{\mathfrak{a}}^{i}(M)\right)\right\}\left(\operatorname{resp} . \quad \lambda_{\mathfrak{a}}^{\mathfrak{b}}(M)=\inf \left\{\operatorname{depth} M_{\mathfrak{p}}+\operatorname{ht}(\mathfrak{a}+\mathfrak{p}) / \mathfrak{p} \mid \mathfrak{p} \in \operatorname{Spec} R \backslash \operatorname{Var}(\mathfrak{b})\right\}\right)$ is the $\mathfrak{b}$-finiteness dimension of $M$ relative to $\mathfrak{a}$ (resp. the $\mathfrak{b}$-minimum $\mathfrak{a}$-adjusted depth of $M)$, see [4, Definitions 9.1.5 and 9.2.2].

Recently, Khashyarmanesh and Salarian in [9], gave a very elegant generalization of the Faltings' Annihilator Theorem over Gorenstein rings. It is well-known that

$$
\begin{aligned}
f_{\mathfrak{a}}^{\mathfrak{b}}(M) & =\inf \left\{i \in \mathbb{N}_{0} \mid \mathfrak{b}^{t} H_{\mathfrak{a}}^{i}(M) \neq 0 \text { for all } t \in \mathbb{N}_{0}\right\} \\
& =\inf \left\{i \in \mathbb{N}_{0} \mid \operatorname{dim} \operatorname{Supp} \mathfrak{b}^{t} H_{\mathfrak{a}}^{i}(M) \geq 0 \text { for all } t \in \mathbb{N}_{0}\right\}
\end{aligned}
$$

Key words and phrases. Annihilation theorem, Finiteness dimension, Local cohomology.

2010 Mathematics Subject Classification: 13D45, 14B15, 13E05.

This research was been in part supported by a grant from IPM.

*Corresponding author: e-mail: naghipour@ipm.ir (Reza Naghipour). 
and

$$
\begin{aligned}
\lambda_{\mathfrak{a}}^{\mathfrak{b}}(M) & =\inf \left\{\lambda_{\mathfrak{a} R_{\mathfrak{p}}}^{\mathfrak{b} R_{\mathfrak{p}}}\left(M_{\mathfrak{p}}\right) \mid \mathfrak{p} \in \operatorname{Spec} R\right\} \\
& =\inf \left\{\lambda_{\mathfrak{a} R_{\mathfrak{p}}}^{\mathfrak{b} R_{\mathfrak{p}}}\left(M_{\mathfrak{p}}\right) \mid \operatorname{dim} R / \mathfrak{p} \geq 0\right\} .
\end{aligned}
$$

Now, for a non-negative integer $n$, we define the $n$th $\mathfrak{b}$-finiteness dimension of $M$ relative to $\mathfrak{a}$ (resp. the $n$th $\mathfrak{b}$-minimum $\mathfrak{a}$-adjusted depth of $M$ ) by

$$
f_{\mathfrak{a}}^{\mathfrak{b}}(M)_{n}:=\inf \left\{i \in \mathbb{N}_{0} \mid \operatorname{dim} \operatorname{Supp}^{t} H_{\mathfrak{a}}^{i}(M) \geq n \text { for all } t \in \mathbb{N}_{0}\right\}
$$

(resp.

$$
\left.\lambda_{\mathfrak{a}}^{\mathfrak{b}}(M)_{n}=: \inf \left\{\lambda_{\mathfrak{a} R_{\mathfrak{p}}}^{\mathfrak{b} R_{\mathfrak{p}}}\left(M_{\mathfrak{p}}\right) \mid \operatorname{dim} R / \mathfrak{p} \geq n\right\}\right) .
$$

Note that $f_{\mathfrak{a}}^{\mathfrak{b}}(M)_{n}$ and $\lambda_{\mathfrak{a}}^{\mathfrak{b}}(M)_{n}$ are either positive integers or $\infty$ and that $f_{\mathfrak{a}}^{\mathfrak{b}}(M)_{0}=$ $f_{\mathfrak{a}}^{\mathfrak{b}}(M)$ and $\lambda_{\mathfrak{a}}^{\mathfrak{b}}(M)_{0}=\lambda_{\mathfrak{a}}^{\mathfrak{b}}(M)$. So it is rather natural to ask whether Faltings' Annihilator Theorem, as stated in above, generalizes in the obvious way to the invariants $f_{\mathfrak{a}}^{\mathfrak{b}}(M)_{n}$ and $\lambda_{\mathfrak{a}}^{\mathfrak{b}}(M)_{n}$. More precisely, as a main result of this paper, we prove the following:

Theorem 1.1. Assume that $R$ is a homomorphic image of a Gorenstein ring. Let $\mathfrak{a}$ and $\mathfrak{b}$ be ideals of $R$ such that $\mathfrak{b} \subseteq \mathfrak{a}$, and let $M$ be a finitely generated $R$-module. Then, for every non-negative integer $n$,

$$
f_{\mathfrak{a}}^{\mathfrak{b}}(M)_{n}=\lambda_{\mathfrak{a}}^{\mathfrak{b}}(M)_{n}
$$

The result in Theorem 1.1 is proved in Theorem 2.14. Our method is based on the theory of Gorenstein dimensions or $G$-dimension. One of our tools for proving Theorem 1.1 is the following, which will play a key role in this paper.

Proposition 1.2. Assume that $R$ is a Gorenstein ring. Let $\mathfrak{a}$ and $\mathfrak{b}$ be ideals of $R$ such that $\mathfrak{b} \subseteq \mathfrak{a}$, and let $M$ be a finitely generated $R$-module. Then, for every non-negative integer $n$,

$$
f_{\mathfrak{a}}^{\mathfrak{b}}(M)_{n}=\lambda_{\mathfrak{a}}^{\mathfrak{b}}(M)_{n}
$$

Throughout this paper, $R$ will always be a commutative Noetherian ring with non-zero identity, $\mathfrak{a}, \mathfrak{b}$ will denote ideals of $R$ and $M$ will denote a finitely generated $R$-module. For any ideal $\mathfrak{a}$ of $R$, we denote $\{\mathfrak{p} \in \operatorname{Spec} R: \mathfrak{p} \supseteq \mathfrak{a}\}$ by $V(\mathfrak{a})$. Also, for any ideal $\mathfrak{b}$ of $R$, the radical of $\mathfrak{b}$, denoted by $\operatorname{Rad}(\mathfrak{b})$, is defined to be the set $\left\{x \in R: x^{n} \in \mathfrak{b}\right.$ for some $\left.n \in \mathbb{N}\right\}$. For any unexplained notation and terminology concerning Gorenstein dimensions we refer the reader to [5].

\section{Faltings' Annihilator Theorem}

The main goal of this section is to provide a generalization of the Faltings' Theorem for the annihilation of local cohomology modules over a Gorenstein ring. The main results are Theorems 2.10 and 2.14. Firstly, let us, introduce the concept of Gorenstein dimension. The notion of Gorenstein dimension ( $G$-dimension) was introduced by Auslander [1] and 
was deeply studied by him and Bridger [2]. For an $R$-module $L$ the biduality map is the canonical $R$-homomorphism

$$
\delta_{L}: L \longrightarrow \operatorname{Hom}_{R}\left(\operatorname{Hom}_{R}(L, R), R\right),
$$

defined by $\delta_{L}(x)(f)=f(x)$ for all $f \in \operatorname{Hom}_{R}(L, R)$ and $x \in L$.

Definition 2.1. (cf. [5, 1.1.2]) A finitely generated $R$-module $M$ belongs to the $G$-class $G(R)$ if and only if

(1) $\operatorname{Ext}_{R}^{i}(M, R)=0$ for all $i>0$;

(2) $\operatorname{Ext}_{R}^{i}\left(\operatorname{Hom}_{R}(M, R), R\right)=0$ for all $i>0$;

(3) The biduality map $\delta_{M}: M \longrightarrow \operatorname{Hom}_{R}\left(\operatorname{Hom}_{R}(M, R), R\right)$ is an isomorphism.

Definition 2.2. (cf. [5, 1.2.1]) A G-resolution of a finitely generated $R$-module $M$ is a sequence of modules in $G(R)$,

$$
\cdots \longrightarrow G_{l} \longrightarrow G_{l-1} \longrightarrow \cdots \longrightarrow G_{2} \longrightarrow G_{1} \longrightarrow G_{0} \longrightarrow 0,
$$

which is exact at $G_{l}$ for all $l>0$ and has $G_{0} / \operatorname{Im}\left(G_{1} \longrightarrow G_{0}\right) \simeq M$. That is, there is an exact sequence

$$
\cdots \longrightarrow G_{l} \longrightarrow G_{l-1} \longrightarrow \cdots \longrightarrow G_{2} \longrightarrow G_{1} \longrightarrow G_{0} \longrightarrow M \longrightarrow 0 .
$$

The resolution is said to be of finite length $n$ if $G_{n} \neq 0$ and $G_{l}=0$ for all $l>n$.

Note that every finitely generated $R$-module has a resolution by finitely generated free modules and, thereby, a $G$-resolution.

Definition 2.3. (cf. [5, 1.2.3]) A finitely generated $R$-module $M$ is said to have finite $G$-dimension, if it has a $G$-resolution of finite length. We set Gdim0 $=-\infty$ and for $M \neq 0$, we define $G$-dimension of $M$ as follows: for any positive integer $n$, we say that $M$ has $G$-dimension at most $n$, and write $\operatorname{Gdim}_{R} M \leqslant n$ if and only if $M$ has a $G$-resolution of length n. If $M$ has no $G$-resolution of finite length, then we say that it has infinite $G$-dimension and write $\operatorname{Gdim}_{R} M=\infty$.

Corollary 2.4. A commutative Noetherian local ring $R$ is Gorenstein if and only if every finitely generated $R$-module has finite $G$-resolution.

Proof. See [7, Corollary 2].

Remark 2.5. Let $M$ be a finitely generated $R$-module. For each $t \in \mathbb{N}_{0} \cup\{-\infty\}$, put $C_{t}(M)=\left\{\mathfrak{p} \in \operatorname{Spec} R \mid \operatorname{Gdim} M_{\mathfrak{p}}>t\right\}$ and $c_{t}(M)=\bigcap_{\mathfrak{p} \in C_{t}(M)} \mathfrak{p}$. Then $C_{t}(M)$ is a closed subset of Spec $R$ (in the Zariski topology) and

$$
\sqrt{0: M}=c_{-\infty}(M) \subseteq c_{0}(M) \subseteq c_{1}(M) \subseteq \cdots \subseteq c_{t}(M) \subseteq \cdots .
$$

We now state and prove some preliminary lemmas and a proposition which help us to conclude the main results.

Lemma 2.6. Let $M$ be a finitely generated $R$-module, and let $\mathfrak{p} \in \operatorname{Spec} R$ be such that $\operatorname{Gdim} M_{\mathfrak{p}}<\infty$. Then there exists $s \in R \backslash \mathfrak{p}$ such that, for every proper ideal $\mathfrak{a}$ of $R$, we have

$$
s H_{\mathfrak{a}}^{i}(M)=0 \quad \text { for all } i<\operatorname{grade}(\mathfrak{a}, R)-\operatorname{Gdim} M_{\mathfrak{p}} .
$$


Proof. Set $h:=\operatorname{Gdim} M_{\mathfrak{p}}$. We use induction on $h$. If $h=-\infty$, then $M_{\mathfrak{p}}=0$ and the result is clear from [4, Lemma 9.4.1]. When $h=0$, the desired result follows from [9, Lemma 2.9]. We therefore assume, inductively, that $h>0$ and the result has been proved for smaller values of $h$. There is a non-zero, finitely generated free $R$-module $F$ and an exact sequence

$$
0 \longrightarrow N \longrightarrow F \longrightarrow M \longrightarrow 0
$$

of $R$-modules and $R$-homomorphisms. Localization yields an exact sequence

$$
0 \longrightarrow N_{\mathfrak{p}} \longrightarrow F_{\mathfrak{p}} \longrightarrow M_{\mathfrak{p}} \longrightarrow 0 \text {. }
$$

Therefore, in view of [5, Corollary 1.2.9(c)], $\operatorname{Gdim} N_{\mathfrak{p}}=h-1$ and so, by inductive hypothesis, there exists $s \in R \backslash \mathfrak{p}$ such that, for every proper ideal $\mathfrak{a}$ of $R$, we have

$$
s H_{\mathfrak{a}}^{i}(N)=0 \quad \text { for all } i<\operatorname{grade}(\mathfrak{a}, R)-h+1 .
$$

Thus $s H_{\mathfrak{a}}^{i+1}(N)=0$ for all $i<\operatorname{grade}(\mathfrak{a}, R)-h$. Let $\mathfrak{a}$ be a proper ideal of $R$ and let $i \in \mathbb{N}_{0}$ with $i<\operatorname{grade}(\mathfrak{a}, R)-h$. Now, in view of the exact sequence

$$
H_{\mathfrak{a}}^{i}(F) \longrightarrow H_{\mathfrak{a}}^{i}(M) \longrightarrow H_{\mathfrak{a}}^{i+1}(N)
$$

and [4, Lemma 6.2.7], we have $s H_{\mathfrak{a}}^{i}(M)=0$. This completes the inductive step.

Lemma 2.7. Let $M$ be a finitely generated $R$-module, and $t \in \mathbb{N}_{0} \cup\{-\infty\}$. Then there exists $n \in \mathbb{N}$ such that, for every proper ideal $\mathfrak{a}$ of $R$, we have

$$
c_{t}(M)^{n} H_{\mathfrak{a}}^{i}(M)=0 \quad \text { for all } i<\operatorname{grade}(\mathfrak{a}, R)-t .
$$

Proof. Let $\mathfrak{p} \in U:=\operatorname{Spec} R \backslash C_{t}(M)$. Thus Gdim $M_{\mathfrak{p}} \leqslant t$. By Lemma 2.6, there exists $s_{\mathfrak{p}} \in R \backslash \mathfrak{p}$ such that, for every proper ideal $\mathfrak{a}$ of $R$, we have $s H_{\mathfrak{a}}^{i}(M)=0$ for all $i<\operatorname{grade}(\mathfrak{a}, R)-\operatorname{Gdim} M_{\mathfrak{p}}$. Set $\mathfrak{g}:=\sum_{\mathfrak{p} \in U} s_{\mathfrak{p}} R$, and observe that, for every proper ideal $\mathfrak{a}$ of $R$, we have $\mathfrak{g} H_{\mathfrak{a}}^{i}(M)=0$ for all $i<\operatorname{grade}(\mathfrak{a}, R)-t$. As $c_{t}(M) \subseteq \sqrt{\mathfrak{g}}$, there exists $n \in \mathbb{N}$ such that $c_{t}(M)^{n} \subseteq \mathfrak{g}$, and the result now follows from this.

Proposition 2.8. Let $R$ be a Gorenstein ring, let $M$ be a finitely generated $R$-module, and let $\mathfrak{a}, \mathfrak{b}$ be ideals of $R$ such that $\mathfrak{b} \subseteq \mathfrak{a}$. Then, for all $\mathfrak{q} \in V(\mathfrak{a})$ with $\operatorname{dim} R / \mathfrak{q} \geq n$,

$$
\mathfrak{b} R_{\mathfrak{q}} \subseteq c_{\text {ht } \mathfrak{q}-\lambda_{\mathfrak{a}}^{\mathfrak{b}}(M)_{n}}\left(M_{\mathfrak{q}}\right)
$$

Proof. Let $\mathfrak{q} \in \operatorname{Var}(\mathfrak{a})$ with $\operatorname{dim} R / \mathfrak{q} \geq n$ and let $\mathfrak{p}$ be an arbitrary prime ideal of $R$ such that $\mathfrak{p} R_{\mathfrak{q}} \in C_{\mathrm{ht} \mathfrak{q}-\lambda_{\mathfrak{a}}^{\mathfrak{b}}(M)_{n}}\left(M_{\mathfrak{q}}\right)$. It is enough to show that $\mathfrak{b} R_{\mathfrak{q}} \subseteq \mathfrak{p} R_{\mathfrak{q}}$. To achieve this, suppose that the contrary is true, i.e., $\mathfrak{b} R_{\mathfrak{q}} \nsubseteq \mathfrak{p} R_{\mathfrak{q}}$, and look for a contradiction. Then it follows from [4, Remarks 9.2.3] that

$$
\infty>\operatorname{depth} M_{\mathfrak{p}}+\operatorname{ht}\left(\mathfrak{q} R_{\mathfrak{q}}+\mathfrak{p} R_{\mathfrak{q}}\right) / \mathfrak{p} R_{\mathfrak{q}} \geq \lambda_{\mathfrak{q} R_{\mathfrak{q}}}^{\mathfrak{b} R_{\mathfrak{q}}}\left(M_{\mathfrak{q}}\right) \geq \lambda_{\mathfrak{a} R_{\mathfrak{q}}}^{\mathfrak{b} R_{\mathfrak{q}}}\left(M_{\mathfrak{q}}\right) \geq \lambda_{\mathfrak{a}}^{\mathfrak{b}}(M)_{n}
$$

Next, since $R_{\mathfrak{q}}$ is catenary, it yields that

$$
\text { ht } \mathfrak{q}-\text { ht } \mathfrak{p}=\text { ht } \mathfrak{q} R_{\mathfrak{q}}-\text { ht } \mathfrak{p} R_{\mathfrak{q}}=\text { ht } \mathfrak{q} R_{\mathfrak{q}} / \mathfrak{p} R_{\mathfrak{q}}=\text { ht }\left(\mathfrak{q} R_{\mathfrak{q}}+\mathfrak{p} R_{\mathfrak{q}}\right) / \mathfrak{p} R_{\mathfrak{q}}
$$


Also, as $R_{\mathfrak{p}}$ is Gorenstein, it follows from Auslander-Birdger formula (see [5, Theorem 1.4.8] and Corollary 2.4, that

$$
\operatorname{depth} M_{\mathfrak{p}}+\operatorname{Gdim} M_{\mathfrak{p}}=\operatorname{depth} R_{\mathfrak{p}}=\text { ht } \mathfrak{p} .
$$

Consequently

$$
\text { ht } \mathfrak{p}-\operatorname{Gdim} M_{\mathfrak{p}}+\text { ht } \mathfrak{q}-\operatorname{ht} \mathfrak{p} \geq \lambda_{\mathfrak{a}}^{\mathfrak{b}}(M)_{n},
$$

and so

$$
\operatorname{Gdim}\left(M_{\mathfrak{q}}\right)_{\mathfrak{p} R_{\mathfrak{q}}}=\operatorname{Gdim} M_{\mathfrak{p}} \leqslant \operatorname{ht} \mathfrak{q}-\lambda_{\mathfrak{a}}^{\mathfrak{b}}(M)_{n} .
$$

Therefore $\mathfrak{p} R_{\mathfrak{q}} \notin C_{\mathrm{ht} \mathfrak{q}-\lambda_{\mathfrak{a}}^{\mathfrak{b}}(M)_{n}}\left(M_{\mathfrak{q}}\right)$, which is a contradiction.

Lemma 2.9. Let $L \longrightarrow M \longrightarrow N$ be an exact sequence of $R$-homomorphisms and $R$ modules. Suppose that $n, t$ and $s$ be non-negative integers such that $\operatorname{dim} \operatorname{Supp}\left(\mathfrak{b}^{t} L\right)<$ $n$ and $\operatorname{dim} \operatorname{Supp}\left(\mathfrak{b}^{s} N\right)<n$. Then there exists a non-negative integer $l$ such that $\operatorname{dim} \operatorname{Supp}\left(\mathfrak{b}^{l} M\right)<n$.

Proof. Set $l:=t+s$. It is enough to show that for each $\mathfrak{p} \in \operatorname{Spec} R$ with $\operatorname{dim} R / \mathfrak{p} \geq n$, we have

$$
\left(\mathfrak{b}^{l} M\right)_{\mathfrak{p}}=\left(\mathfrak{b} R_{\mathfrak{p}}\right)^{l} M_{\mathfrak{p}}=0 .
$$

To do this, let $m \in M_{\mathfrak{p}}$ and we consider the exact sequence

$$
L_{\mathfrak{p}} \stackrel{f}{\longrightarrow} M_{\mathfrak{p}} \stackrel{g}{\longrightarrow} N_{\mathfrak{p}},
$$

of $R_{\mathfrak{p}}$-modules and $R_{\mathfrak{p}}$-homomorphisms. Now, for each $u \in \mathfrak{b}^{s} R_{\mathfrak{p}}$ we have $g(u m)=$ $u g(m)=0$, and so $u m \in \operatorname{Ker} g=\operatorname{Im} f$. Thus there exists $v \in L_{\mathfrak{p}}$ such that $u m=f(v)$. Also, for each $w \in \mathfrak{b}^{t} R_{\mathfrak{p}}$, we have

$$
w u m=w f(v)=f(w v)=0 .
$$

Hence $\mathfrak{b}^{l} R_{\mathfrak{p}} m=\left(\mathfrak{b}^{t} R_{\mathfrak{p}}\right)\left(\mathfrak{b}^{s} R_{\mathfrak{p}}\right) m=0$, and therefore $\left(\mathfrak{b} R_{\mathfrak{p}}\right)^{l} M_{\mathfrak{p}}=0$, as required.

We are now ready to state and prove the generalization of the Faltings' Theorem for the annihilation of local cohomology modules over a Gorenstein ring.

Theorem 2.10. (Faltings' Annihilator Theorem) Assume that $R$ is a Gorenstein ring and $M$ a finitely generated $R$-module. Let $\mathfrak{a}$ and $\mathfrak{b}$ be ideals of $R$ such that $\mathfrak{b} \subseteq \mathfrak{a}$. Then, for every non-negative integer $n$,

$$
f_{\mathfrak{a}}^{\mathfrak{b}}(M)_{n}=\lambda_{\mathfrak{a}}^{\mathfrak{b}}(M)_{n} .
$$

Proof. Let $f_{\mathfrak{a}}^{\mathfrak{b}}(M)_{n}=h$. Then there exists a non-negative integer $t$ such that for all integers $i<h$ we have,

$$
\operatorname{dim} \operatorname{Supp}\left(\mathfrak{b}^{t} H_{\mathfrak{a}}^{i}(M)\right)<n .
$$

Thus $\left(\mathfrak{b}^{t} H_{\mathfrak{a}}^{i}(M)\right)_{\mathfrak{p}}=0$ for all $i<h$ and for all $\mathfrak{p} \in \operatorname{Spec} R$ with $\operatorname{dim} R / \mathfrak{p} \geq n$, and so $f_{\mathfrak{a} R_{\mathfrak{p}}}^{\mathfrak{b} R_{\mathfrak{p}}}\left(M_{\mathfrak{p}}\right) \geq h$ for all $\mathfrak{p} \in \operatorname{Spec} R$ with $\operatorname{dim} R / \mathfrak{p} \geq n$. Hence, on use of [4, Theorem 9.3.5], 
we have

$$
\begin{aligned}
f_{\mathfrak{a}}^{\mathfrak{b}}(M)_{n}=h & \leqslant \inf \left\{f_{\mathfrak{a} R_{\mathfrak{p}}}^{\mathfrak{b} R_{\mathfrak{p}}}\left(M_{\mathfrak{p}}\right) \mid \mathfrak{p} \in \operatorname{Spec} R \text { and } \operatorname{dim} R / \mathfrak{p} \geq n\right\} \\
& \leqslant \inf \left\{\lambda_{\mathfrak{a} R_{\mathfrak{p}}}^{\mathfrak{b} R_{\mathfrak{p}}}\left(M_{\mathfrak{p}}\right) \mid \mathfrak{p} \in \operatorname{Spec} R \text { and } \operatorname{dim} R / \mathfrak{p} \geq n\right\}=\lambda_{\mathfrak{a}}^{\mathfrak{b}}(M)_{n} .
\end{aligned}
$$

Now, for proving the inequality $f_{\mathfrak{a}}^{\mathfrak{b}}(M)_{n} \geq \lambda_{\mathfrak{a}}^{\mathfrak{b}}(M)_{n}$, there are two cases to consider.

Case 1. Suppose that $\operatorname{dim} R / \mathfrak{a} \leq n$. Then the set

$$
\mathcal{T}:=\{\mathfrak{p} \mid \mathfrak{p} \in V(\mathfrak{a}) \text { and } \operatorname{dim} R / \mathfrak{p}=n\},
$$

is finite. Let $\mathcal{T}=\left\{\mathfrak{p}_{1}, \ldots, \mathfrak{p}_{h}\right\}$ and consider an integer $j$ with $1 \leqslant j \leqslant h$. Set $t_{j}=$ ht $\mathfrak{p}_{j}-\lambda_{\mathfrak{a}}^{\mathfrak{b}}(M)_{n}$. Since ht $\mathfrak{p}_{j}=\operatorname{grade}\left(\mathfrak{p}_{j} R_{\mathfrak{p}_{j}}, R_{\mathfrak{p}_{j}}\right)$, we can deduce from Lemma 2.7 that there exists $s_{j} \in \mathbb{N}$ such that

$$
c_{t_{j}}\left(M_{\mathfrak{p}_{j}}\right)^{s_{j}} H_{\mathfrak{p}_{j} R_{\mathfrak{p}_{j}}}^{i}\left(M_{\mathfrak{p}_{j}}\right)=0 \quad \text { for all } i<\operatorname{ht} \mathfrak{p}_{j}-t_{j}=\lambda_{\mathfrak{a}}^{\mathfrak{b}}(M)_{n} .
$$

Moreover, in view of Proposition 2.8, $\mathfrak{b} R_{\mathfrak{p}_{j}} \subseteq c_{t_{j}}\left(M_{\mathfrak{p}_{j}}\right)$. Set $t=\max \left\{s_{1}, \ldots, s_{h}\right\}$. Then

$$
\left(\mathfrak{b}^{t} H_{\mathfrak{a}}^{i}(M)\right)_{\mathfrak{p}_{j}}=\left(\left(\mathfrak{b} R_{\mathfrak{p}_{j}}\right)^{t} H_{\mathfrak{a}_{\mathfrak{p}_{j}}}^{i}\left(M_{\mathfrak{p}_{j}}\right)\right) \subseteq c_{t_{j}}\left(M_{\mathfrak{p}_{j}}\right)^{t} H_{\mathfrak{p}_{j} R_{\mathfrak{p}_{j}}}^{i}\left(M_{\mathfrak{p}_{j}}\right)=0 \quad \text { for all } i<\lambda_{\mathfrak{a}}^{\mathfrak{b}}(M)_{n} .
$$

Hence $\mathfrak{p}_{1}, \ldots, \mathfrak{p}_{h} \notin \operatorname{Supp}\left(\mathfrak{b}^{t} H_{\mathfrak{a}}^{i}(M)\right)$, and so $\operatorname{dim} \operatorname{Supp}\left(\mathfrak{b}^{t} H_{\mathfrak{a}}^{i}(M)\right) \leqslant n-1$. Therefore $f_{\mathfrak{a}}^{\mathfrak{b}}(M)_{n} \geq \lambda_{\mathfrak{a}}^{\mathfrak{b}}(M)_{n}$, as required.

Case 2. Now, suppose that $\operatorname{dim} R / \mathfrak{a}>n$ and we show that $f_{\mathfrak{a}}^{\mathfrak{b}}(M)_{n} \geq \lambda_{\mathfrak{a}}^{\mathfrak{b}}(M)_{n}$. Suppose, the contrary, that $f_{\mathfrak{a}}^{\mathfrak{b}}(M)_{n}<\lambda_{\mathfrak{a}}^{\mathfrak{b}}(M)_{n}$ and look for a contradiction. To this end, as $R$ is Noetherian, we can (and do) assume that $\mathfrak{a}$ is a maximal element of the set

$$
\Sigma:=\left\{\mathfrak{a}^{\prime} \in \mathscr{I}(R) \mid \text { there exists } \mathfrak{b}^{\prime} \in \mathscr{I}(R) \text { with } \mathfrak{b}^{\prime} \subseteq \mathfrak{a}^{\prime} \text { and } f_{\mathfrak{a}^{\prime}}^{\mathfrak{b}^{\prime}}(M)_{n}<\lambda_{\mathfrak{a}^{\prime}}^{\mathfrak{b}^{\prime}}(M)_{n}\right\},
$$

where $\mathscr{I}(R)$ denotes the set of all ideals of $R$. Let $\mathfrak{p}_{1}, \ldots, \mathfrak{p}_{h}$ be the distinct minimal primes of $\mathfrak{a}$. Then there is an integer $i$ with $1 \leq i \leq h \operatorname{such}$ that $\operatorname{dim} R / \mathfrak{a}=\operatorname{dim} R / \mathfrak{p}_{i}$. By [4, Exercise 9.4.9] and Proposition [2.8, we have

$$
\mathfrak{b} R_{\mathfrak{p}_{i}} \subseteq c_{\left(\mathrm{ht} \mathfrak{p}_{i}-\lambda_{\mathfrak{a}}^{\mathfrak{b}}(M)_{n}\right)}\left(M_{\mathfrak{p}_{i}}\right)=\left(c_{\left(\mathrm{ht} \mathfrak{p}_{i}-\lambda_{\mathfrak{a}}^{\mathfrak{b}}(M)_{n}\right)}(M)\right)_{\mathfrak{p}_{i}} .
$$

Therefore there exists $u \in R \backslash \mathfrak{p}_{i}$ such that $\mathfrak{b} R_{u} \subseteq\left(c_{\left(\mathrm{ht} \mathfrak{p}_{i}-\lambda_{\mathfrak{a}}^{\mathfrak{b}}(M)_{n}\right)}(M)\right)_{u}$. As $\operatorname{dim} R / \mathfrak{p}_{i}>0$, there exists $\mathfrak{q} \in \operatorname{Spec} R$ such that $\mathfrak{p}_{i} \varsubsetneqq \mathfrak{q}$. Let $v \in \mathfrak{q} \backslash \mathfrak{p}_{i}$ and $w \in \cap_{j \neq i} \mathfrak{p}_{j} \backslash \mathfrak{p}_{i}$. Set $s:=u v w$. Then in view of [4, Exercise 9.4.9], we have

$$
\mathfrak{b} R_{s} \subseteq\left(c_{\left(\mathrm{ht} \mathfrak{p}_{i}-\lambda_{\mathfrak{a}}^{\mathfrak{b}}(M)_{n}\right)}(M)\right)_{s}=c_{\left(\mathrm{ht} \mathfrak{p}_{i} R_{s}-\lambda_{\mathfrak{a}}^{\mathfrak{b}}(M)_{n}\right)}\left(M_{s}\right)=c_{\left(\mathrm{ht} \mathfrak{a} R_{s}-\lambda_{\mathfrak{a}}^{\mathfrak{b}}(M)_{n}\right)}\left(M_{s}\right) .
$$

Since grade $\left(\mathfrak{a} R_{s}, R_{s}\right)=$ ht $\mathfrak{a} R_{s}$, it follows from Lemma 2.7 that there exists $t_{1} \in \mathbb{N}$ such that

$$
\left(c_{\left(\mathrm{ht} \mathfrak{a} R_{s}-\lambda_{\mathfrak{a}}^{\mathfrak{b}}(M)_{n}\right)}\left(M_{s}\right)\right)^{t_{1}} H_{\mathfrak{a} R_{s}}^{i}\left(M_{s}\right)=0 \quad \text { for all } i<\lambda_{\mathfrak{a}}^{\mathfrak{b}}(M)_{n} .
$$

Hence $\mathfrak{b}^{t_{1}} H_{\mathfrak{a} R_{s}}^{i}\left(M_{s}\right)=0$ for all $i<\lambda_{\mathfrak{a}}^{\mathfrak{b}}(M)_{n}$. Therefore, by virtue of [4, Theorem 4.2.1], we have $\mathfrak{b}^{t_{1}} H_{\mathfrak{a}}^{i}\left(M_{s}\right)=0$ for all $i<\lambda_{\mathfrak{a}}^{\mathfrak{b}}(M)_{n}$. Since $\mathfrak{a} \varsubsetneqq \mathfrak{a}+R s \varsubsetneqq R$, it follows by the 'maximality' assumption on $\mathfrak{a}$ that $\lambda_{\mathfrak{a}+R s}^{\mathfrak{b}}(M)_{n} \leq f_{\mathfrak{a}+R s}^{\mathfrak{b}}(M)_{n}$. Now, as in view of 
[4, Remark 9.2.3], $\lambda_{\mathfrak{a}}^{\mathfrak{b}}(M)_{n} \leq \lambda_{\mathfrak{a}+R s}^{\mathfrak{b}}(M)_{n}$, it follows that there exists $t_{2} \in \mathbb{N}$ such that $\operatorname{dim} \operatorname{Supp}\left(\mathfrak{b}^{t_{2}} H_{\mathfrak{a}+R s}^{i}(M)\right) \leq n-1$ for all $i<\lambda_{\mathfrak{a}}^{\mathfrak{b}}(M)_{n}$. By the exact sequence

$$
\cdots \longrightarrow H_{\mathfrak{a}+R s}^{i}(M) \longrightarrow H_{\mathfrak{a}}^{i}(M) \longrightarrow H_{\mathfrak{a}}^{i}\left(M_{s}\right) \longrightarrow \cdots
$$

and Lemma 2.9, it follows that there exists $t \in \mathbb{N}$ such that $\operatorname{dim} \operatorname{Supp}\left(\mathfrak{b}^{t} H_{\mathfrak{a}}^{i}(M)\right) \leq n-1$ for all $i<\lambda_{\mathfrak{a}}^{\mathfrak{b}}(M)_{n}$. Therefore $\lambda_{\mathfrak{a}}^{\mathfrak{b}}(M)_{n} \leq f_{\mathfrak{a}}^{\mathfrak{b}}(M)_{n}$, and this contradiction completes the proof.

Lemma 2.11. Let $f: R \longrightarrow R^{\prime}$ be a surjective homomorphism of Noetherian rings and let $f^{*}:$ Spec $R^{\prime} \longrightarrow$ Spec $R$ be the induced map. Then for any $R^{\prime}$-module $L$,

$$
\operatorname{Supp}_{R} L=f^{*}\left(\operatorname{Supp}_{R^{\prime}} L\right) \text {. }
$$

Proof. Follows easily from [10, Proposition 3.2] and [3, Ch. IV, Sec. 3, Proposition 7].

Lemma 2.12. Let $f: R \longrightarrow R^{\prime}$ be a surjective homomorphism of Noetherian rings, and $\mathfrak{a}$ and $\mathfrak{b}$ be ideals of $R$ such that $\mathfrak{b} \subseteq \mathfrak{a}$. Let $M^{\prime}$ be a finitely generated $R^{\prime}$-module. Then

$$
f_{\mathfrak{a}}^{\mathfrak{b}}\left(M^{\prime}\right)_{n}=f_{\mathfrak{a} R^{\prime}}^{\mathfrak{b} R^{\prime}}\left(M^{\prime}\right)_{n} .
$$

Proof. Let $f^{*}:$ Spec $R^{\prime} \longrightarrow$ Spec $R$ be the induced map. Then, for each $t \in \mathbb{N}_{0}$, we have

$$
f^{*}\left(\operatorname{Supp}_{R^{\prime}}\left(\mathfrak{b} R^{\prime}\right)^{t} H_{\mathfrak{a} R^{\prime}}^{i}\left(M^{\prime}\right)\right)=\operatorname{Supp}_{R}\left(\mathfrak{b} R^{\prime}\right)^{t} H_{\mathfrak{a} R^{\prime}}^{i}\left(M^{\prime}\right)=\operatorname{Supp}_{R} \mathfrak{b}^{t} H_{\mathfrak{a}}^{i}\left(M^{\prime}\right),
$$

and so $\operatorname{dim}_{R^{\prime}}\left(\mathfrak{b} R^{\prime}\right)^{t} H_{\mathfrak{a} R^{\prime}}^{i}\left(M^{\prime}\right)=\operatorname{dim}_{R} \mathfrak{b}^{t} H_{\mathfrak{a}}^{i}\left(M^{\prime}\right)$. Thus

$$
f_{\mathfrak{a}}^{\mathfrak{b}}\left(M^{\prime}\right)_{n}=f_{\mathfrak{a} R^{\prime}}^{\mathfrak{b} R^{\prime}}\left(M^{\prime}\right)_{n},
$$

as required.

Before we state Theorem 2.14 which is the main result of this paper, we give a couple of lemmas that in the proof of Theorem 2.14.

Lemma 2.13. Let $\mathfrak{a}$ and $\mathfrak{b}$ be ideals of $R$ such that $\mathfrak{b} \subseteq \mathfrak{a}$. Let $M$ be a finitely generated $R$-module, and let $\mathfrak{c}$ be an ideal of $R$ such that $\mathfrak{c} \subseteq(0: M)$. Then

$$
\lambda_{\mathfrak{a}}^{\mathfrak{b}}(M)_{n}=\lambda_{(\mathfrak{a}+\mathfrak{c}) / \mathfrak{c}}^{(\mathfrak{b}+\mathfrak{c})}(M)_{n}
$$

Proof. In view of [4, Lemma 9.2.6] we have

$$
\begin{aligned}
\lambda_{\mathfrak{a}}^{\mathfrak{b}}(M)_{n} & =\inf \left\{\lambda_{\mathfrak{a} R_{\mathfrak{p}}}^{\mathfrak{b} R_{\mathfrak{p}}}\left(M_{\mathfrak{p}}\right) \mid \mathfrak{p} \in \operatorname{Spec} R \text { and } \operatorname{dim} R / \mathfrak{p} \geq n\right\} \\
& =\inf \left\{\lambda_{\left(\mathfrak{a} R_{\mathfrak{p}}+\mathfrak{c} R_{\mathfrak{p}}\right) / \mathfrak{c} R_{\mathfrak{p}}}^{\left(\mathfrak{b} R_{\mathfrak{p}}+\mathfrak{c} R_{\mathfrak{p}}\right) / \mathfrak{c} R_{\mathfrak{p}}}\left(M_{\mathfrak{p}}\right) \mid \mathfrak{p} \in \operatorname{Spec} R \text { and } \operatorname{dim} R / \mathfrak{p} \geq n\right\} \\
& =\inf \left\{\lambda_{((\mathfrak{a}+\mathfrak{c}) / \mathfrak{c})_{\mathfrak{p} / \mathfrak{c}}}^{((\mathfrak{c}+\mathfrak{c}}\left(M_{\mathfrak{p} / \mathfrak{c}}\right) \mid \mathfrak{p} / \mathfrak{c} \in \operatorname{Spec} R / \mathfrak{c} \text { and } \operatorname{dim}(R / \mathfrak{c}) /(\mathfrak{p} / \mathfrak{c}) \geq n\right\} \\
& =\lambda_{(\mathfrak{a}+\mathfrak{c}) / \mathfrak{c}}^{(\mathfrak{b} \mathfrak{c}) / \mathfrak{c}}(M)_{n},
\end{aligned}
$$

as required. 
We are now ready to state and prove the main result of this paper which is a generalization of the Faltings' Theorem for the annihilation of local cohomology modules whenever the ring $R$ is a homomorphic image of a Noetherian Gorenstein ring.

Theorem 2.14. Assume that $R$ is a homomorphic image of a Gorenstein ring. Let $\mathfrak{a}$ and $\mathfrak{b}$ be ideals of $R$ such that $\mathfrak{b} \subseteq \mathfrak{a}$, and let $M$ be a finitely generated $R$-module. Then, for each $n \in \mathbb{N}_{0}$,

$$
f_{\mathfrak{a}}^{\mathfrak{b}}(M)_{n}=\lambda_{\mathfrak{a}}^{\mathfrak{b}}(M)_{n}
$$

Proof. By assumption there is a Gorenstein ring $R^{\prime}$ and a surjective homomorphism of Noetherian rings $f: R^{\prime} \longrightarrow R$. Let $\mathfrak{a}^{\prime}$ and $\mathfrak{b}^{\prime}$ be ideals of $R^{\prime}$ such that $\mathfrak{a}=\mathfrak{a}^{\prime} R$ and $\mathfrak{b}=\mathfrak{b}^{\prime} R$. Then by Lemmas 2.12, 2.13, and Theorem 2.10,

$$
f_{\mathfrak{a}}^{\mathfrak{b}}(M)_{n}=f_{\mathfrak{a}^{\prime} R}^{\mathfrak{b}^{\prime} R}(M)_{n}=f_{\mathfrak{a}^{\prime}}^{\mathfrak{b}^{\prime}}(M)_{n}=\lambda_{\mathfrak{a}^{\prime}}^{\mathfrak{b}^{\prime}}(M)_{n}=\lambda_{\mathfrak{a}}^{\mathfrak{b}}(M)_{n},
$$

as required.

\section{Acknowledgments}

The authors would like to thank Professor Hossein Zakeri for his reading of the first draft and valuable discussions. Also, we would like to thank from School of Mathematics, Institute for Research in Fundamental Sciences (IPM), for its financial support.

\section{REFERENCES}

[1] M. Auslander, Anneaux de Gorenstein et torsion en algèbre commutative, Séminaire d'algèbre commutative $1966 / 67$.

[2] M. Auslander and M. Bridger, Stable module theory, Mem. Amer. Math. Soc., 94 (1969).

[3] N. Bourbaki, Commutative algebra, Addison- Wesley, 1972.

[4] M.P. Brodmann and R.Y. Sharp, Local cohomology; an algebraic introduction with geometric applications, Cambridge University Press, Cambridge,1998.

[5] L. W. Christensen, Gorenstein dimensions, Lecture Notes in Mathematics, no. 1747, Springer-Verlag, Berlin, 2000.

[6] G. Faltings, Über die Annulatoren lokaler Kohomologiegruppen, Arch. Math. 30 (1978) 473-476.

[7] S. Goto, Vanishing of $\operatorname{Ext}_{\mathrm{R}}^{\mathrm{i}}(\mathrm{M}, \mathrm{A})$, J. Math. Kyoto Univ. 22 (3) (1982) 481-484.

[8] A. Grothendieck, Local cohomology, Notes by R. Hartshorne, Lecture Notes in Math., 862 (Springer, New York, 1966).

[9] K. Khashyarmanesh and Sh. Salarian, Faltings, theorem for the annihilation of local cohomology modules over a Gorenstein ring, Proc. Amer. Math. Soc. 132(2004), 2215-2220.

[10] L. Melkersson, On asymptotic stability for sets of prime ideals connected with the powers of an ideal, Math. Proc. Camb. Phil. Soc. 107(1990), 267-271.

Department of Mathematics, University of Tabriz, Tabriz, Iran; and School of Mathematics, Institute for Research in Fundamental Sciences (IPM), P.O. Box 19395-5746, TEHRAN, IRAN.

E-mail address: naghipour@ipm.ir

E-mail address: naghipour@tabrizu.ac.ir

E-mail address: m_doustimehr@tabrizu.ac.ir 